\title{
Sonoendocrinology and Monitoring Assisted Reproduction Technology
}

\author{
${ }^{1}$ Sonal Panchal, ${ }^{2}$ Chaitanya Nagori
}

\begin{abstract}
Reproductive function in a human being consists of continuous hormonal changes in the female. These hormonal changes are responsible for dynamic changes occurring in the ovarian and uterine morphology and vascularity. Ultrasound and Doppler are very useful modalities for assessing these changes, and therefore, very effective for diagnosis of hormonal derangements and monitoring the assisted reproductive technology (ART) treated cycles. Among the common hormones that need to be assessed, androgen can be correlated with high antral follicle count (AFC) and high uterine artery resistance and anti-Müllerian hormone (AMH) also has a positive correlation with AFC. Follicle-stimulating hormone (FSH) is reflected as follicular growth, and luteinizing hormone ( $\mathrm{LH}$ ) by ovarian stromal echogenicity and vascularity and endometrial hyperechogenicity. Estrogen adequacy can be judged by follicular and endometrial flow in preovulatory phase whereas progesterone adequacy can be judged by corpus luteal and endometrial flow in mid luteal phase. Day-to-day hormonal assessment can therefore be replaced by ultrasound and Doppler during ART.
\end{abstract}

Keywords: Doppler, Ovarian stromal vascularity, Sonoendocrinal correlation.

How to cite this article: Panchal S, Nagori C. Sonoendocrinology and Monitoring Assisted Reproduction Technology. Donald School J Ultrasound Obstet Gynecol 2016;10(4):376-386.

Source of support: Nil

Conflict of interest: None

\section{INTRODUCTION}

Reproductive function in a human being consists of continuous hormonal changes in the female. These hormonal changes are responsible for dynamic changes occurring in the ovarian and uterine morphology and vascularity. These morphological and vascular changes can be best studied by transvaginal ultrasound and Doppler. Correlating the morphological and vascular changes with their physiological and hormonal basis can be a helpful way to understand the hormonal changes occurring during the menstrual cycle whether natural or stimulated. These changes can be monitored by different imaging modalities.

\footnotetext{
${ }^{1}$ Consultant, ${ }^{2}$ Director and Consultant

${ }^{1}$ Department of Ultrasound, Dr. Nagori's Institute for Infertility and IVF, Ahmedabad, Gujarat, India

${ }^{2}$ Department of Infertility, Dr. Nagori's Institute for Infertility and IVF, Ahmedabad, Gujarat, India
}

Corresponding Author: Sonal Panchal, Consultant Department of Ultrasound, Dr. Nagori's Institute for Infertility and IVF, Ahmedabad, Gujarat, India, e-mail: sonalyogesh@yahoo.com

\section{IMAGING MODALITIES FOR ASSESSMENT OF REPRODUCTIVE SYSTEM}

Imaging modalities that can study the morphological and vascular changes of ovary, endometrium, and uterus are ultrasound and Doppler. Soon after the ultrasound was used for antenatal scanning, it was also used to assess the nonpregnant uterus and ovaries. Transabdominal ultrasound was the approach used earlier but this approach has certain inherent disadvantages. It uses a lowfrequency probe that has a low resolution. Moreover, the probe is placed far from the pelvic organs, which further reduces the precision of information available on scan. Apart from this, the full bladder that patient requires to hold is also a discomfort.

With the development of endocavity probes, transvaginal route became very popular for scanning the pelvic organs, uterus, ovaries, and fallopian tubes. It uses a highfrequency probe, and the probe is placed very close to the uterus and ovaries, both help to achieve high-resolution images of uterus and ovaries. The resolution was further improved in high-end scanners by advanced features that are used to improve the $b$ mode image. These are harmonics, speckle reduction imaging, and compound resolution imaging. All these together made it possible to assess morphology of uterus and ovaries more precisely. Improvement in the Doppler sensitivity with advancing technology made it possible to assess the blood flow changes in small blood vessels of ovarian stroma, follicle, and endometrium. Volume ultrasound further leads to assessment of global vascularity of ovary, follicle, and endometrium. Moreover, these volume studies are also used to assess the volumes of the organs or structures instead of just diameters. Magnetic resonance imaging (MRI) though allows detailed morphological assessment, but is less patient-friendly and more expensive than ultrasound. The greater disadvantage of MRI is that it is not a dynamic examination and also cannot be repeatedly done to observe day-to-day changes. This disadvantage can be over powered by volume ultrasound and also by the real-time studies that are possible by ultrasound and not by MRI.

Ultrasound has been widely used for the assessment of pathologies of uterus, fallopian tubes, and ovaries. But here, we shall discuss its role for understanding the physiology, in assessment and monitoring of natural and 
stimulated menstrual cycles respectively, and will try to understand and correlate the hormonal changes with the ultrasound findings of ovaries and uterus in different phases of menstrual cycle.

The hormones that play a major role in the reproductive physiology are:

- Androgen

- Anti-Müllerian hormone (AMH)

- Follicle-stimulating hormone (FSH)

- Luteinizing hormone (LH)

- Estrogen

- Progesterone.

\section{ANDROGEN}

The menstrual cycle starts on first day of the menstruation. But actually the recruitment of the follicles from preantral to antral follicles starts in the late luteal phase of the previous cycle. The antral follicles are $2 \mathrm{~mm}$ and more in size and are then recognizable by ultrasound. Recruitment of these follicles is a function of androgen, and antral follicle count (AFC) represents the functional ovarian reserve. Higher androgen level would lead to more follicles being converted from preantral to antral, and so higher AFC. ${ }^{1}$ It is for this reason that patients with polycystic ovary syndrome (PCOS), who have hyperandrogenemia, show higher AFC as compared with controls (Figs 1A and B). Obesity and insulin resistance may enhance follicular excess by dysregulation of $\mathrm{AMH}$ through pathway of hyperandrogenemia. ${ }^{2}$

Antral follicle count and ovarian volume showed significant correlation with $\mathrm{AMH}$, total testosterone, and free androgen index. ${ }^{2}$ Hyperinsulinemic PCOS patients had an increased vascularity of the ovarian stroma. ${ }^{3}$ Increased ovarian stromal blood flow in PCOS may be because of over expression of vascular endothelial growth factor, which modulates the permeability of theca cells and increase insulin-like growth factor $1{ }^{4,5}$ This in turn enhances gonadotropin-stimulated steroid production in granulosa cells and theca cells, resulting in increased ovarian androgen production and subsequently increased AMH. ${ }^{6}$
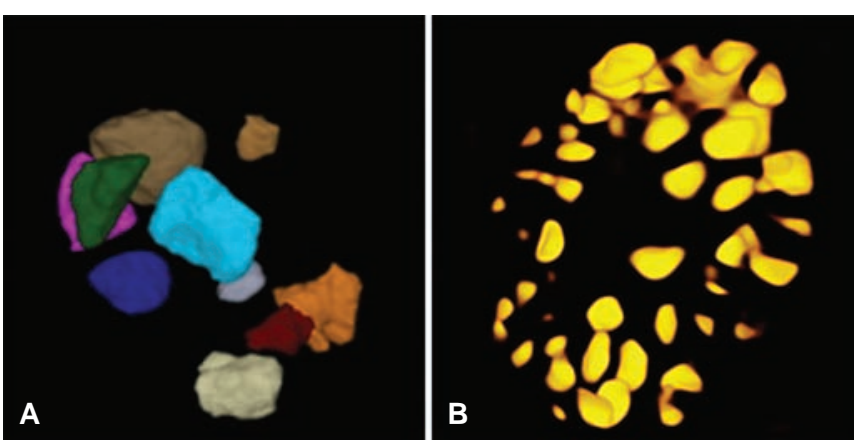

Figs 1A and B: (A) Low antral follicle count; (B) high antral follicle count (on inversion mode 3D rendered image)
Moreover, androgen is responsible for smooth muscle tone. Uterine arteries have thick muscularis layer to allow the flexibility and dynamicity in its caliber during different phases of menstruation and also during pregnancy and labor. This layer consists of smooth muscle fibers and therefore androgen leads to increased tone in these muscles and therefore high resistance flow in uterine arteries in PCOS patients, during the whole cycle (Fig. 2). ${ }^{7}$

The ovarian stroma/total ovarian volume ratio was the most accurate predictor of both hyperandrogenemia (area under the curve, 0.915; p <0.0001) and hirsutism (area under the curve, $0.891 ; \mathrm{p}<0.0001)^{8}$

High androgen is reflected as:

- More antral follicles

- Increased uterine artery resistance

- Stromal predominance.

\section{ANTI-MÜLLERIAN HORMONE}

Biochemically ovarian reserve can be assessed by $\mathrm{AMH}$, FSH, and inhibin B.

Anti-Müllerian hormone is produced by granulosa cells of preantral and small antral follicles of between 2 and $6 \mathrm{~mm}$, before they become sensitive to FSH. It is a regulator of recruitment of follicles. Anti-Müllerian hormone has an advantage that it can be in any phase of cycle, though threshold in both phases may be different.

We have been all relating AFCs to AMH. But it is worth keeping in mind that androgen is the cause for the recruitment of the antral follicles and $\mathrm{AMH}$ is a result of recruited antral follicles. Antral follicle count and ovarian volume showed significant correlation with AMH.

The number of small antral follicles $(2-6 \mathrm{~mm})$ is significantly related to age and also, independent of age, to all endocrine ovarian reserve tests (ORTs), suggesting the number of small antral follicles represents the functional ovarian reserve. ${ }^{9}$

Several studies have shown that AMH and AFC are both almost equally accurate tests for assessment of ovarian reserve. Antral follicle count and ovarian volume

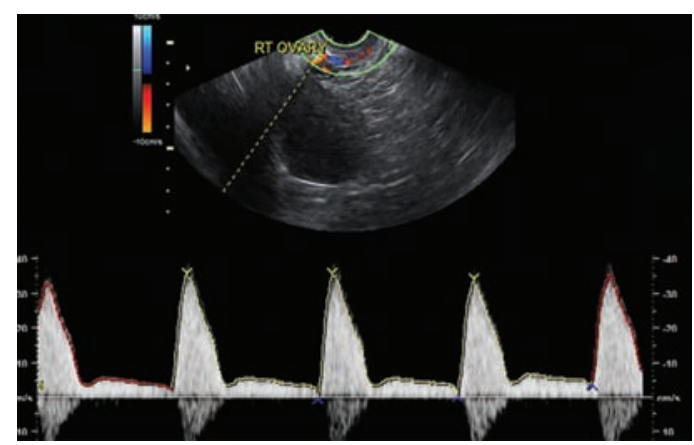

Fig. 2: High uterine artery resistance 
provide direct measurements of ovarian reserve, while $\mathrm{AMH}$, inhibin $\mathrm{B}$, and estradiol are released from growing follicles and so their levels reflect the size of developing follicle cohort. ${ }^{10}$

Serum AMH levels have been shown to strongly correlate with the number of antral follicles ${ }^{11,12}$ and have appeared to be cycle independent. ${ }^{13,14}$ The receiver operative characteristic (ROC) curves do not suggest a clearly better predictive ability for AMH than for AFC, and the difference was not statistically significant $(\mathrm{p}=$ 0.73). This implies that the best poor response predictor to date, $\mathrm{AMH}^{15}$ has obtained company from a test that may have some crucial advantages.

Sensitivity and specificity for $\mathrm{AMH}$ were 82 and $76 \%$ respectively, and 82 and $80 \%$ respectively, for AFC. Comparison of the summary estimates and ROC curves for $\mathrm{AMH}$ and $\mathrm{AFC}$ showed no statistical difference. Both $\mathrm{AMH}$ and AFC are accurate predictors of excessive response to ovarian hyperstimulation. Moreover, both tests appear to have clinical value. ${ }^{16}$ Small AFC and $\mathrm{AMH}$ have similar predictive accuracy for high ovarian response with area under curve of 0.961 and 0.922 respectively. The sensitivity and specificity for prediction of high ovarian response were 89 and $92 \%$ for small AFC and 93 and $78 \%$ for $\mathrm{AMH}$ at the cutoff values of $\geq 16$ and $\geq 34.5$ pmol/L respectively. ${ }^{17}$ In our study we compared the efficacy of AFC and AMH in PCO and non-PCO group. The results showed correlation of AFC and follicles $>12 \mathrm{~mm}$ on day of human chorionic gonadotropin (hCG) in PCO group was 0.56 and non-PCO group was 0.63 and for AMH and follicles $>12 \mathrm{~mm}$ on day of hCG in PCO group was 0.42 , and non-PCO group was 0.47. Correlation of AFC with number of ova retrieved on OPU in PCO group is 0.44 and for non-PCO group is 0.50 . The value for $\mathrm{AMH}$ is 0.39 in $\mathrm{PCO}$ and 0.43 for non-PCO group. (Significance of correlation at 0.01 level is required to be higher than 0.283). ${ }^{18}$

In a meta-analysis by Broer et $\mathrm{al}^{19}{ }^{19}$ it has been shown that $\mathrm{AMH}$ has at least the same level of accuracy and

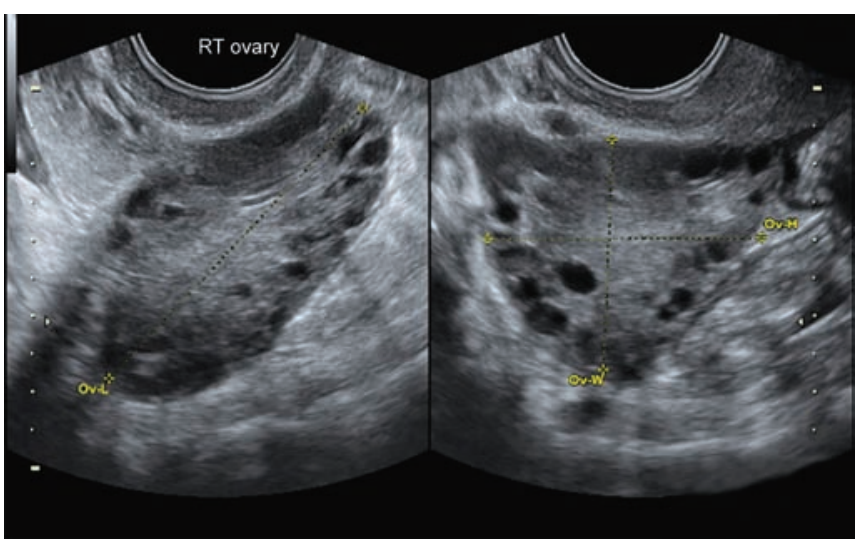

Fig. 3: Small antral follicles clinical value for the prediction of poor response and nonpregnancy as AFC.

Anti-Müllerian hormone can be closely correlated with AFC (Fig. 3) and can also be used as a test for ovarian reserve instead of $\mathrm{AMH}$, without compromising the accuracy and efficacy.

\section{FOLLICLE-STIMULATING HORMONE}

Follicle-stimulating hormone is produced by pituitary and its secretion is controlled by the negative feedback of estrogen and also acts on granulosa cells to produce estrogen. In the initial phases of the menstrual cycle, the estrogen level is low, the FSH level is a little high, whereas in the preovulatory phase, the estrogen level rises and there is a fall in the FSH level. When the follicle size is less than 6 $\mathrm{mm}$, these follicles are FSH independent. At a size of 6 to $9 \mathrm{~mm}$, these become FSH sensitive by the development of $\mathrm{FSH}$ receptors, and beyond $9 \mathrm{~mm}$ these follicles are $\mathrm{FSH}$ dependent (Fig. 4). Anti-Müllerian hormone, a product of preantral and small antral follicles, inhibits factors affecting FSH sensitivity of the follicle. As AMH levels decrease with follicle growth, this inhibition would be removed. ${ }^{20}$ This means that the follicular growth beyond $6 \mathrm{~mm}$ size suggests FSH action. Therefore, faster growth of the follicles indicates high FSH. If this growth is fast in early follicular phase, it indicates high basal FSH. Early follicular recruitment-follicle of larger than $10 \mathrm{~mm}$ on the baseline scan indicates high basal FSH levels. But it is important to remember that the follicle that is, recruited early may grow fast on stimulation, but is less likely to produce good quality follicle due to its shorter duration to maturity.

Similarly, in stimulated cycle, too high doses of FSH may lead to faster follicular growth than normal which is 2 to $3 \mathrm{~mm} /$ day. This is not an advantage again as the ovum maturity may lag behind the size of the follicle in these cases; moreover, endometrial growth also may not cope up with this growth in follicular size.

Whereas a slow follicular growth rate indicates that the FSH level is lower than optimum. This is the situation in POCS patients. Because of multiple follicular development and aromatization of excess androgen to estrogen, there is rise in estrogen level leading to initiation of negative feedback to FSH, slow rise in FSH, and therefore, slow growth of follicles.

Follicle-stimulating hormone dynamics can be represented by ultrasound findings as follows:

High basal FSH - early recruitment of follicle (Fig. 5).

High doses of FSH - faster growth of the follicle than normal.

Slow rise in FSH or FSH of inadequate bioactivity (which is the case in aging ovaries): Slow growth rate of follicle. 


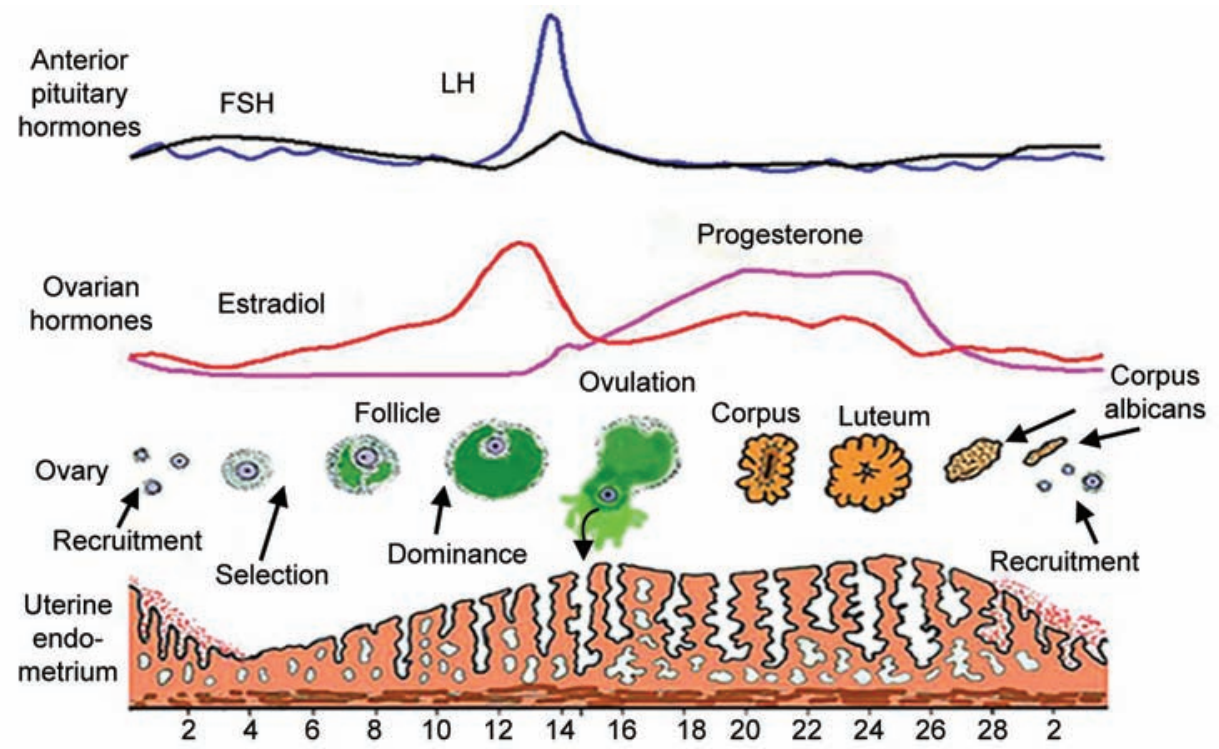

Fig. 4: Diagrammatic representation of hormonal and morphological changes occurring during the menstrual cycle

\section{LUTEINIZING HORMONE}

Luteinizing hormone is also produced by pituitary. It is controlled by positive feedback of estrogen and acts on theca cells to produce progesterone from cholesterol. Luteinizing hormone is also responsible for proliferation of theca cells.

Elevated LH levels may be responsible for increased stromal vascularization due to neoangiogenesis, catecholaminergic stimulation, and leukocyte and cytokine activation. This means LH is responsible for stromal vascularity. ${ }^{21}$ This is the reason why PCOS patients have increased stromal vascularity as compared with the normal controls.

The vascular parameters of the ovarian stromal vessels in PCOS and normal patients are as follows ${ }^{22}$ (Fig. 6) (Table 1):

When assessed by 3D power Doppler, compared with the normal controls, women with PCOS had higher AFC

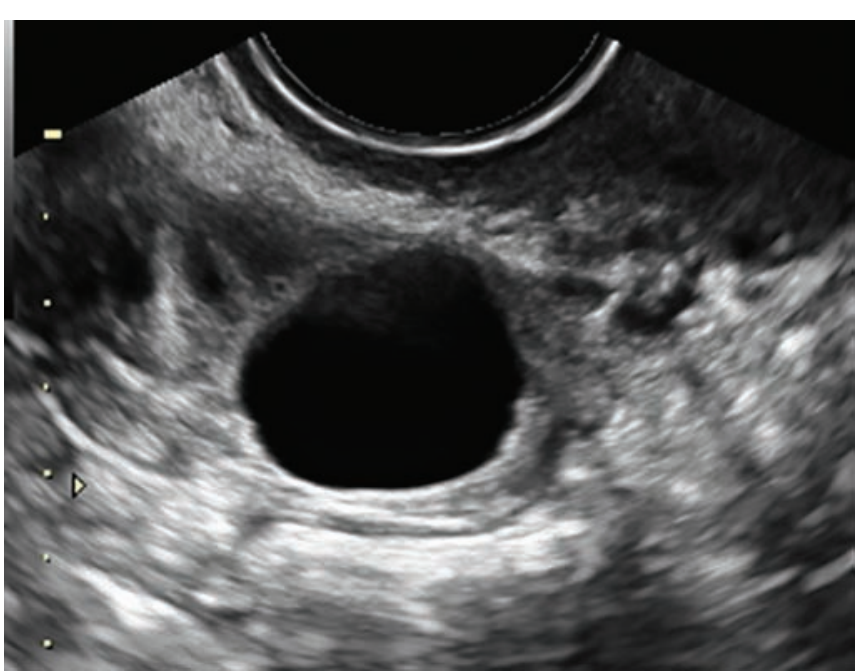

Fig. 5: Day 3 scan showing dominant follicle: Early follicular recruitment (median 16.3 vs 5.5 per ovary), ovarian volume (12.56 vs $5.6 \mathrm{~mL}$ ), stromal volume (10.79 vs $4.69 \mathrm{~mL}$ ), and stromal vascularization (VI 3.85 vs $2.79 \%$, VFI 1.27 vs 0.85 ), though this study quotes that 2D power Doppler indices were not higher in PCOS than in controls. ${ }^{23}$

\section{What Is the Effect of High LH in PCOS?}

It is known that PCOS is not a cause but a result of chronic anovulation. It is a process of evolution from adolescent aged multicystic ovaries to polycystic ovaries. As a result of marginally high androgen levels in early follicular phase in polycystic ovarian disease patients, there is recruitment of multiple antral follicles. But these follicles grow only up to 6 to $7 \mathrm{~mm}$ under the effect of androgen.

Table 1: Vascular parameters of ovarian stromal vessels in PCOS and normal patients

\begin{tabular}{lll}
\hline Parameter & PCOS & Normal \\
\hline Resistance index (RI) & $0.54 \pm 0.04$ & $0.78 \pm 0.06$ \\
Pulsatility index (PI) & $0.89 \pm 0.04$ & $1.87 \pm 0.38$ \\
Peak systolic velocity (PSV) & $11.9 \pm 3.2$ & $9.6 \pm 2.1$ \\
\hline
\end{tabular}

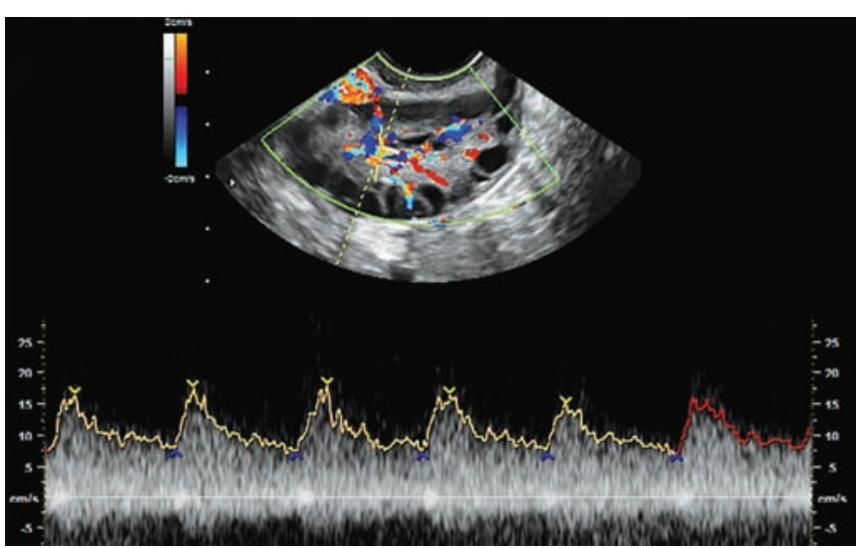

Fig. 6: Stromal vascularity in polycystic ovary 
These small follicles do secret estrogen, and therefore higher estrogen causes a negative feedback for FSH. Low FSH is not enough for follicular maturation and leads to chronic anovulation. But all these follicles do not become dominant due to high LH and/or AMH levels. These follicles which were recruited but not matured undergo atresia, their granulosa cells get converted into theca cells and contribute to stroma. This stroma tries to accommodate itself in the ovarian capsule and therefore starts becoming dense initially and then increases the ovarian volume. Polycystic ovarian morphology has been found to be a better discriminator than ovarian volume between PCOS and control women. ${ }^{24}$ Patients having long-standing PCOS and long-standing anovulation have more dense stroma, and this cardinal feature has been shown to be the presence of a bright, highly echogenic stroma on transvaginal ultrasound. ${ }^{25}$ In a study by Franks et al, ${ }^{26}$ it is also shown that PCO morphology in normal women is not a morphological variant of normal ovaries, but rather represent a functional entity. Lam and Raine-Fenning have concluded in their study that the current criteria will fail to identify a group of ovulatory, normo-androgenic women still at risk of complications classically associated with PCOS, such as ovarian hyperstimulation syndrome, failed implantation, miscarriage, and hyperinsulinemia. To identify these women, further information, particularly about the ovarian stroma and the degree of vascularization, is required. ${ }^{27}$

It is known according to two-cell two-hormone theory ${ }^{28,29}$ that LH is responsible for conversion of granulosa cells to theca cells. In the preovulatory phase, this action of LH is seen whenever any follicle is exposed to LH. This means that if a mature follicle is exposed to LH, it leads to conversion of the granulosa cells of this follicle into theca cells and progresses it toward rupture, leading to corpus luteal formation. Instead, if an immature follicle i.e., dominant is exposed to LH, the granulosa cells of this follicle are converted into theca cells, and the follicle is converted into a luteinized unruptured follicle (LUF), which produces progesterone but not as much as corpus luteum, and if a follicle which is still not dominant, may be only an antral follicle, the follicle undergoes atresia and the theca cells of this follicle also contribute to stroma.

This shows that detecting the time of natural LH surge or the time when surrogate surge is to be given is the key to success of any assisted reproductive technology (ART). This can be decided by follicle maturity.

\section{Ultrasound Parameters of Follicle with Varying Estrogen Levels}

Follicular maturity is assessed biochemically by estrogen level, and on ultrasound by b mode and Doppler assessment of the follicle. Estrogen is responsible for perifollicular vascularity. As seen in the diagrammatic representation of the hormonal variations during the menstrual cycle, the estrogen level gradually starts rising after the 5 th day of the menstrual cycle, when the dominant follicle is selected. Increase in perifollicular vascularity of dominant follicle in theca layer starts developing as early as 8th day of the cycle. Fall in perifollicular resistance index (RI) starts 2 days before ovulation. ${ }^{30}$ This is the time when the shoot up is seen in the estrogen level. At ovulation when estrogen level has just passed the peak, the RI is the lowest. This means that the RI of the perifollicular vessels can be inversely correlated with the estrogen levels. Increase in peak systolic velocity (PSV) starts 29 hours before ovulation and continues for at least 72 hours after ovulation. ${ }^{31,32}$ This can be explained by the fact that LH is responsible for the influx of blood in the ovary and neoangiogenesis, i.e., essential for the follicular and in turn ovum oxygenation. It is the combined action of estrogen and LH surge that leads to these final circulatory changes before ovulation. Vascular changes at the time of impending ovulation include increased vascularity of the inner wall of the follicle and a coincident surge in blood velocity just prior to eruption. ${ }^{33}$ A marked increase in the PSV around the follicle, in the presence of a relatively constant pulsatility index (PI), could be a sign of follicle maturity and impending ovulation. ${ }^{34}$ In stimulated cycles the surge is surrogate and, i.e., by hCG. The influx of blood flow surrounding the follicle therefore follows hCG.

But it is known that after the peak of LH surge, either natural or surrogate, there is a sudden downfall in the estrogen level, but the rise in the progesterone level is slow and reaches peak only in the mid luteal phase. It is because of this that the uterine artery resistance increases after the LH surge. ${ }^{35,36}$

- High tonic LH levels: Increased ovarian stromal flow on baseline scan

- The LH rise with no dominant follicles: Follicular atresia

- The LH rise with dominant but not mature follicle (functionally): LUF

- Inadequate LH surge with a mature follicle: LUF

- The LH surge with a functionally mature follicle: follicle rupture.

This means a correct time to use the surrogate LH surge (hCG or GnRh agonist) is of utmost importance. And the ultrasound features which would decide the follicular maturity on the pre-hCG scan are as follows (Fig. 7):

- Follicular size of at least $18 \mathrm{~mm}$

- Perifollicular vascularity covering $3 / 4$ th of the follicular circumference ${ }^{37}$

- Perifollicular RI 0.4 to $0.48^{21}$ and PSV $>10 \mathrm{~cm} /$ second. $^{38}$ 

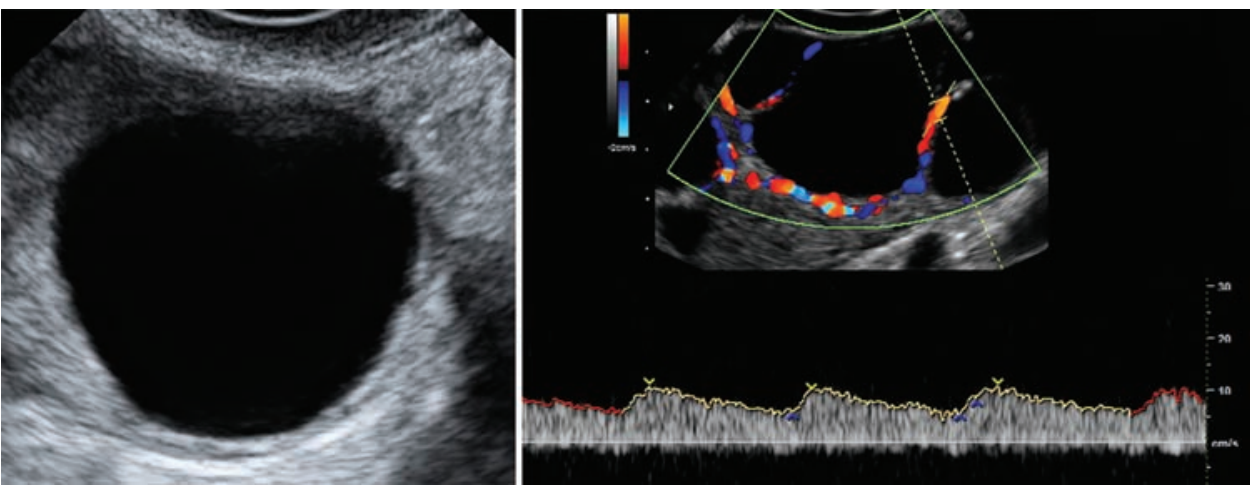

Fig. 7: B mode and Doppler ultrasound parameters of a mature follicle

Follicular RI and PSV are more important in decisionmaking than the size of the follicle. ${ }^{39}$ Ovarian flow correlates well with oocyte recovery rates and hence, may be useful in determining the most appropriate time to administer hCG to optimize recovery rate. Oocytes from severely hypoxic follicles are associated with high frequency of abnormalities of organization of chromosomes on metaphase spindle and may lead to segregation disorders and catastrophic mosaics in embryo. ${ }^{40}$

Change in these parameters may be of help in deciding the time and number of intrauterine inseminations (IUIs). At the LH surge, the perifollicular PSV is $10 \mathrm{~cm} /$ second. According to certain studies, perifollicular PSV goes as high as $45 \mathrm{~cm} /$ second before an hour of ovulation $^{33}$ under the effect of rising LH. This means that if the follicle is said to be functionally mature when PSV is $10 \mathrm{~cm} /$ second, then, i.e., the time when the LH surge starts, and under the effect of that LH, the perifollicular PSV keeps on rising constantly. Rising PSV with steady low RI suggests that LH surge has already established and the follicle is close to rupture. ${ }^{34}$ Steady or decreasing PSV with rising RI suggests that LH surge is either inadequate or has started before the follicle was mature and the follicle is proceeding toward LUF. Therefore, in patients with high pre-hCG perifollicular PSV, early IUI would improve the conception rates.

As the endometrium is exposed to the effect of $\mathrm{LH}$ on the follicles, the outer margin of the endometrium that was sharp like a pencil line earlier, starts becoming fluffy, before it starts becoming hyperechoic.

\section{Preovulatory Findings and LH}

- Fall in perifollicular RI suggests start of LH surge.

- Rise in perifollicular PSV suggests fast rising LH level and impending rupture.

- Rising RI with steady or falling PSV suggests falling or inadequate LH levels and therefore is an indicator of follicle proceeding toward LUF.

- Fluffy outer margins of the endometrium.

\section{ESTROGEN}

It is known that the granulosa cells of follicle are responsible for estrogen secretion, and therefore, increasing follicular size is associated with rising estrogen levels. Therefore, the simplest evidence that the estrogen level is rising is the follicular growth. It is worth while mentioning here that FSH is the cause and estrogen is the result of follicular growth. The pre-hCG perifollicular ultrasound parameters mentioned earlier in this article are the parameters of a follicle, i.e., producing sufficient estrogen. Estrogen is produced by the granulosa cells of the follicle, but uterus is the receptor organ for estrogen. Evidently therefore, assessment of the uterus and endometrium should give the clue to adequacy of estrogen levels and in turn of the functional maturity of the follicle. Increasing endometrial thickness and changing endometrial morphology to multilayered correlates with follicular growth and increasing estrogen levels ${ }^{41}$ (Fig. 8). Cervical mucus (Fig. 9) that was earlier assessed on postcoital test can now be seen on ultrasound as fluid in cervical canal and this again is the indicator of estrogen levels comparable to a mature follicle. Therefore in preovulatory period of the menstrual cycle, multilayered endometrium of at least $6 \mathrm{~mm}$, preferably 8 to $10 \mathrm{~mm}$ in thickness indicates satisfactory estrogen levels and thin endometrium would be an indicator of low estrogen (inadequate follicular maturity). When the estrogen levels are much higher, the endometrium becomes homogenous and isoechoic instead of multilayered. ${ }^{41}$ This is the case in PCOS patients (Fig. 10).

The endometrial thickness can be used as a guide to estrogen levels on the baseline scan also. For the patients on down regulation for in vitro fertilization (IVF), if the baseline scan shows an endometrial thickness of $>3 \mathrm{~mm}$, it is an indicator of inadequate downregulation. ${ }^{42}$ This endometrial thickness usually correlates with 30 to $50 \mathrm{pg} / \mathrm{mL}$ of estrogen. Though in some cases, it may be because of continuing activity of corpus luteum, this may be confirmed by the corpus luteum with pericorpus luteal low-resistance flow in either ovary.

The vascularity of the endometrium also correlates with the estrogen levels. There are several reports by 


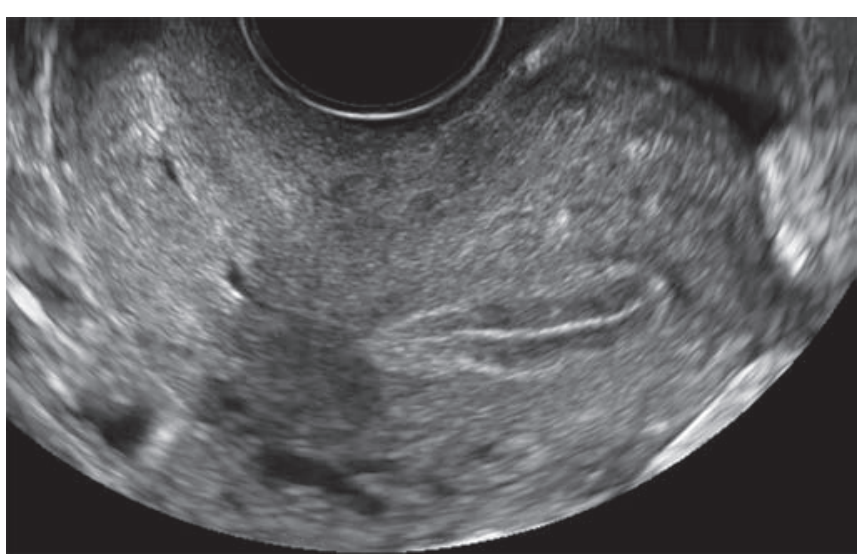

Fig. 8: Multilayered endometrium

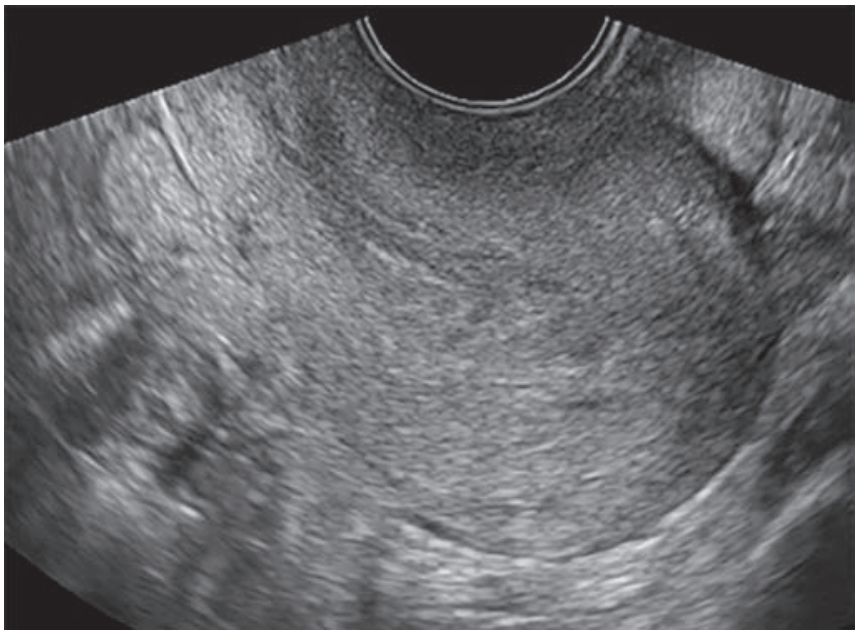

Fig. 10: Homogenously isoechoic-hyperestrinic endometrium

different groups $s^{32,36,43}$ that agree on the fact that implantation rates can be more correlated to the vascularity of the endometrium rather than the thickness and morphology of the endometrium. Endometrial and subendometrial

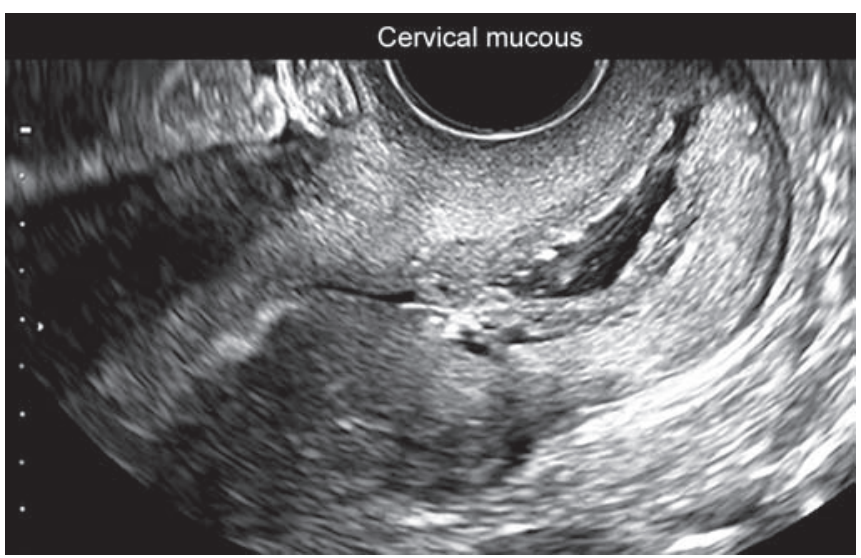

Fig. 9: Cervical mucous

flow increases in the follicular phase and reaches its maximum approximately 3 days prior to ovulation. This is the time when estrogen level starts rising fast, for the final peak. The spiral vessels grow toward the endometrium and penetrate the endometrium. Vascularization of the endometrium is very important for pre-hCG evaluation of the endometrium and implantation. To make its assessment more precise and objective, the vascularity of the endometrium is classified by Applebaum ${ }^{44}$ as follows (Figs 11A to D):

Zone I - Blood vessels reaching endomyometrial junction surrounding the endometrium.

Zone II - Blood vessels reaching outer hyperechoic endometrial edge.

Zone III - Blood vessels reaching intervening endometrial hypoechoic zone.

Zone IV - Blood vessels reaching endometrial cavity: The central line.

Vascularity of the endometrium before ovulation should reach zone 3 to 4 of the endometrium and should
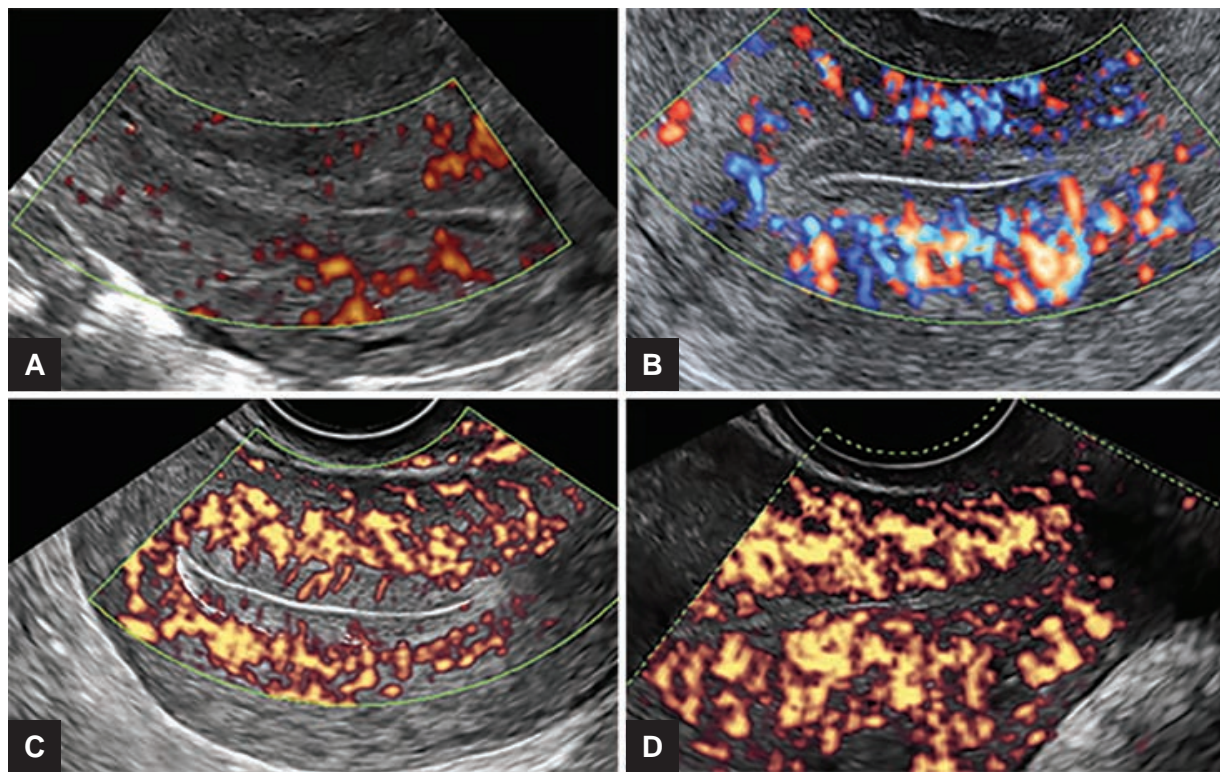

Figs 11A to D: Endometrial vascularity zones: (A) Zone 1, (B) Zone 2, (C) Zone 3, and (D) Zone 4 
cover $5 \mathrm{~mm}^{2}$ area. ${ }^{45}$ The vessels supplying the endometrium should have an RI of $<0.59$. Absence of subendometrial and intraendometrial vascularization on the day of hCG appears to be a useful predictor of failure of implantation in IVF, irrespective of morphological appearance. ${ }^{43}$ Moreover, it has also been observed that when pregnancy is achieved in the absence of endometrial and subendometrial flow on the day of embryo transfer (ET), more than half of these pregnancies will finish as spontaneous miscarriage. ${ }^{46}$

Spiral arteries are branches of uterine arteries and therefore if the resistance in uterine artery is high, evidently resistance in spiral arteries is also high, so assessment of the uterine artery resistance may also be a clue to implantation potential of the endometrium. Just before ovulation the uterine artery resistance rises due to fall in estrogen. hCG administration induces significant increase in the resistance of uterine artery for 48 hours which can affect its evaluation on the day of follicular aspiration/ rupture. ${ }^{35}$ Uterine artery resistance is affected by androgen levels as also discussed earlier, and so in patients with hyperandrogenemia (PCOS), uterine artery resistance remains higher throughout the cycle. This may be one of the reasons for implantation failure or early abortions in patient with PCOS.

It has been shown in several studies that when uterine artery PI > 3.2, implantation rates are extremely low and ET or IUI should be withheld. ${ }^{47-49}$ If the upper limit of cut for uterine artery PI is established at 3 to 3.3, prediction of nonreceptive uterus has specificity of 96 to $100 \%$, positive predictive value of 88 to $100 \%$, sensitivity of 13 to $35 \%$ and negative predictive value of 44 to $56 \%{ }^{50}$

\section{Correlation of Estrogen with Ultrasound Findings}

Thin linear endometrium on baseline scan suggests adequate down regulation and low basal estrogen levels.

A follicle of $18 \mathrm{~mm}$ was thought to indicate a mature follicle and so adequate estrogen levels. But as discussed earlier, it is not the follicle size but the perifollicular blood flow that decides the functional maturity of the follicle and therefore estrogen production.

Conversion of linear endometrium to multilayered endometrium indicates rising estrogen level to achieve the level of LH surge. Multilayered endometrium, i.e., $8 \mathrm{~m}$ or more in thickness indicates adequate estrogen levels for a mature follicle. Homogenization of the multilayered endometrium suggests hyperestrinism as discussed earlier.

\section{SECRETORY PHASE ASSESSMENT}

Rupture of the follicle leads to formation of corpus luteum. Corpus luteum is responsible for progesterone production. The functional efficacy of the corpus luteum can be assessed by Doppler by assessing the pericorpus luteal vascularity.

Segmental uterine and ovarian artery perfusion demonstrates a significant correlation with histological and hormonal markers of uterine receptivity and may aid assessment of luteal phase defect. ${ }^{51}$ A clear correlation between RI of corpus luteum and plasma progesterone levels has been seen in natural cycle. Resistance index of the corpus luteum can, therefore, be used as an adjunct to plasma progesterone assay as an index of luteal function. ${ }^{52}$ A corpus luteum, i.e., functionally normal and produces adequate amount of progesterone shows corpus luteal flow: RI 0.35 to 0.50 and PSV 10 to 15 (Fig. 12).

The receptor organ for progesterone also (like estrogen) is endometrium and its vascular studies can be a reliable clue to adequate progesterone production. Though, endometrial also becomes hyperechoic as a result of progesterone exposure. But soon after rupture of the follicle, the outer margin of the endometrium starts becoming fluffy and blurred. With adequate progesterone levels, that are achieved in the mid-luteal phase, the spiral arteries show RI of 0.48 to 0.52 (low-resistance flow) and uterine artery shows PI of 2.0 to 2.5. This PI is lower than in the preovulatory phase because of smooth muscle relaxing effect of progesterone. Inadequate progesterone production and therefore corpus luteal inadequacy is suggested by high resistance flow in corpus luteal vessels. ${ }^{52}$ Whereas, high spiral artery resistance would suggest inadequate response of endometrium to progesterone. This is because of inadequate progesterone receptors in the endometrium or because of local endometrial cause, like endometrial injury or chronic endometritis.

In luteal phase defect because of low progesterone levels, the resistance in the pericorpus luteal vessels is high. Because of low progesterone levels, there is

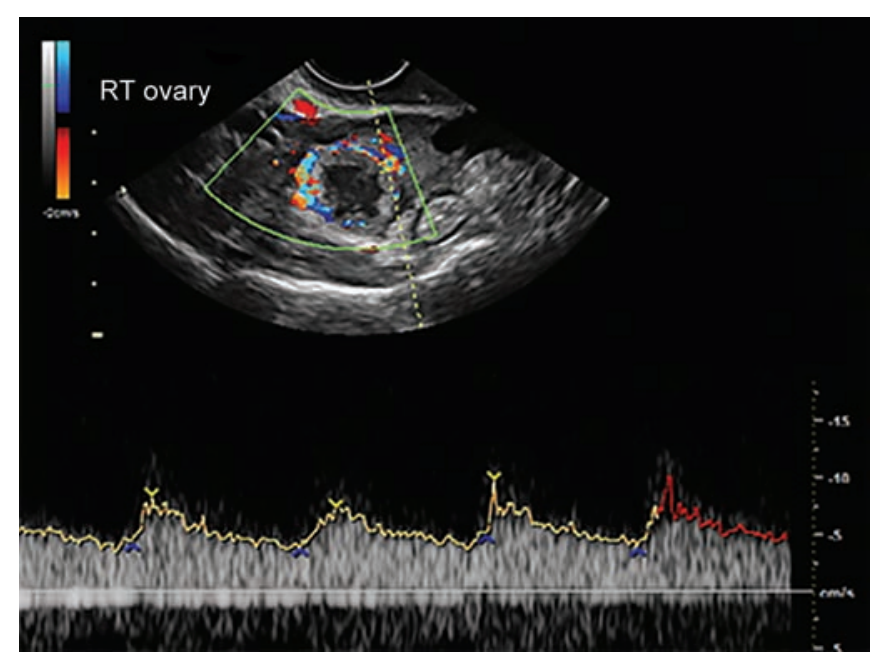

Fig. 12: Normal corpus luteal flow 


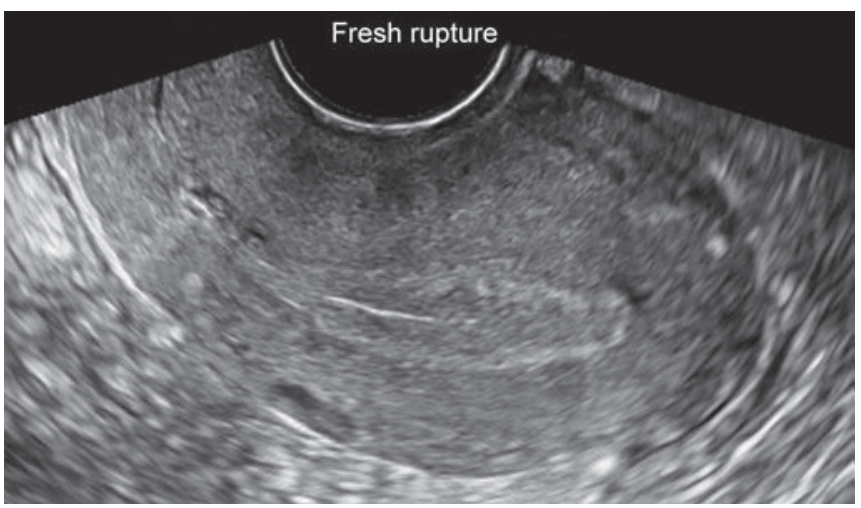

Fig. 13: Early luteal phase endometrium

inadequate relaxation of the muscularis of the uterine artery, and therefore, the uterine artery resistance is high along with higher resistance in its branches - the spiral vessels.

Corpus luteal flow and spiral artery flow in normal cycle and luteal phase defect (Table 2): ${ }^{52}$

Table 2: Secretory phase assessment

\begin{tabular}{lll}
\hline Phase & Normal RI & LPD RI \\
\hline Perifollicular & $0.56 \pm 0.06$ & $0.58 \pm 0.04$ \\
LH peak day (PF) & $0.44 \pm 0.04$ & $0.58 \pm 0.04$ \\
Midluteal phase (CL) & $0.42 \pm 0.06$ & $0.58 \pm 0.04$ \\
Late luteal phase (CL) & $0.50 \pm 0.04$ & $0.58 \pm 0.04$ \\
Periovulatory Endo. & $0.53 \pm 0.04$ & $0.70 \pm 0.06$ \\
Midluteal Endo. & $0.50 \pm 0.02$ & $0.72 \pm 0.06$ \\
Late luteal Endo. & $0.51 \pm 0.04$ & $0.72 \pm 0.04$ \\
\hline
\end{tabular}

CL: Corpus luteum, Endo.: Endometrium

\section{CORRELATION OF PROGESTERONE TO ULTRASOUND FINDINGS}

Blurring of outer margin of multilayered endometrium suggests initiation of progesterone secretion (Fig. 13). Low resistance flow in corpus luteum, with echogenic endometrium (Fig. 14) and low resistance endometrial vascularity in mid luteal phase suggests normal luteal phase. High resistance corpus luteal flow suggests corpus luteal inadequacy. High resistance endometrial flow in mid luteal phase suggests either inadequate progesterone levels or inadequate progesterone receptors in endometrium.

\section{CONCLUSION}

Ultrasound is an excellent tool for assessment of the menstrual cycle. Hormonal changes occurring day to day during the menstrual cycle reflect as morphological and vascular changes in the ovary and the uterus. Assessing these changes by transvaginal ultrasound and Doppler and correctly interpreting can explain the hormonal basis of these changes. Ultrasound with Doppler can thus be used as the only modality for cycle assessment in patients undergoing ART and may be of help to reduce the cost

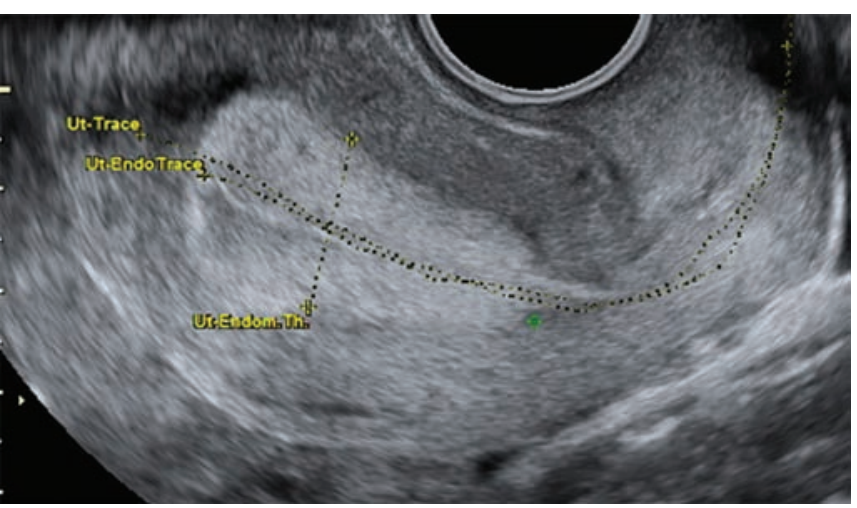

Fig. 14: Secretory endometrium on B mode

of the cycle by avoiding certain hormonal assessments and still maintaining close and accurate watch on the hormonal changes occurring during treatment cycle.

\section{REFERENCES}

1. Jonard S, Robert Y, Cortet-Rudelli C, Decanter C, Dewailly D. Ultrasound examination of polycystic ovaries: is it worth counting the follicles? Hum Reprod 2003 Mar;18(3):598-603.

2. Chen MJ, Yang WS, Chen CL, Wu MY, Yang YS, Ho HN. The relationship between anti-Mullerian hormone, androgen and insulin resistance on the number of antral follicle in women with polycystic ovary syndrome. Hum Reprod 2008 Feb;23(4):952-957.

3. Loverro G, Vicino M, Lorusso F, Vimercati A, Greco P, Selvaggi L. Polycystic ovary syndrome: relationship between insulin sensitivity, sex hormone levels and ovarian stromal blood flow. Gynecol Endocrinol 2001 Apr;15(2):142-149.

4. El Behery MM, Diab AE, Mowafy H, Ebrahiem MA, Shehata AE. Effect of laparoscopic ovarian drilling on vascular endothelial growth factor and ovarian stromal blood flow using 3 dimensional power Doppler. Int J Gynaecol Obstet 2011 Feb;112(2):119-121.

5. Abd El Aal DE, Mohamed SA, Amine AF, Meki AR. Vascular endothelial growth factor and insulin like growth factor-1 in polycystic ovary syndrome and their relationship to ovarian blood flow. Eur J Obstet Gynecol Reprod Biol 2005 Feb;118(2):219-224.

6. Willis DS, Mason HD, Gilling-Smith C, Franks S. Modulation by insulin of follicle stimulating hormone actions in human granulosa cells of normal and polycystic ovaries. J Clin Endocrinol Metab 1996 Jan;81(1):302-309.

7. Ozkan S, Vural B, Caliskan E, Bodur H, Turkoz E, Vural F. Colour Doppler sonographic analysis of uterine and ovarian artery blood flow in women with PCOS. J Clin Ultrasound 2007 Jul-Aug;35(6):305-313.

8. Battaglia C, Battaglia B, Morotti E, Paradisi R, Zanetti I, Meriggiola MC, Venturoli S. Two- and three-dimensional sonographic and color Doppler techniques for diagnosis of polycystic ovary syndrome. The stromal/ovarian volume ratio as a new diagnostic criterion. Ultrasound Med 2012 Jul;31(7):1015-1024.

9. Haadsma ML, Bukman A, Groen H, Roeloffzen EMA, Groenewoud ER, Heineman MJ, Hoek A. The number of small antral follicles $(2-6 \mathrm{~mm})$ determines the outcome of endocrine 
ovarian reserve tests in a subfertile population. Hum Reprod 2007 Jul;22(7):1925-1931.

10. te Velde ER, et al. Advances in fertility studies and reproductive medicine IFFS. 2007;306.

11. Gruijters MJ, Visser JA, Durlinger AL, Themmen AP. Antimullerian hormone and its role in ovarian function. Mol Cell Endocrinol 2003 Dec;211(1-2):85-90.

12. Van Rooij IA, Broekmans FJ, te Velde ER, Fauser BC, Bancsi LF, de Jong FH, Themmen AP. Serum antimullerian hormone levels: a novel measure of ovarian reserve. Hum Reprod 2002 Dec;17(12):3065-3071.

13. Hehenkamp WJ, Looman CW, Themmen AP, de Jong FH, te Velde ER, Broekmans FJ. Antimullerian hormone levels in the spontaneous menstrual cycle do not show substantial fluctuation. J. Clin Endocrinol Metab 2006 Oct;91(10):4057-4063.

14. La MArca A, Stabile G, Artenisio AC, Volpe A. Serum antimullerian hormone throughout the human menstrual cycle. Hum Reprod 2006 Dec;21(12):3103-3107.

15. Hendriks DJ, Mol BW, Bancsi LF, te Velde ER, Broekmans FJ. Antral follicle count in the prediction of poor ovarian response and pregnancy after in vitro fertilization: a meta-analysis and comparison with basal follicle-stimulating hormone level. Fertil Steril 2005 Feb;83:291-301.

16. Broer SL, Dólleman M, Opmeer BC, Fauser BC, Mol BW, Broekmans FJM. AMH and AFC as predictors of excessive response in controlled ovarian hyperstimulation: a metaaanalysis. Hum Reprod 2011;17(1):46-54.

17. Aflatoonian A, Oskouian H, Ahmadi S, Oskouian L. Prediction of high ovarian response to controlled ovarian hyperstimulation: anti-mullerian hormone versus small antral follicle count (2-6mm). J Assist Reprod Genet 2009 Jun;26(6):319-325.

18. Panchal S, Nagori C. Comparison of AFC and AMH for assessment of ovarian reserve. J Hum Reprod Sci 2012 Sep;5(3):274-278.

19. Broer SL, Mol BWJ, Hendriks D, Broekmans FJM. The role of antimullerian hormone in prediction of outcome after IVF: comparison with antral follicle count. Fertil Steril 2009;91(3): 705-714.

20. Pellatt L, Rice S, Dilaver N, Heshri A, Galea R, Brincat M, Brown K, Simpson ER, Mason HD. Anti-Müllerian hormone reduces follicle sensitivity to follicle-stimulating hormone in human granulosa cells. Fertil Steril 2011 Nov;96(5):1246-1251.

21. Battalgia C, Artini PG, Salvatori M, Giulini S, Petraglia F, Maxia N, Volpe A. Ultrasonographic patterns of polycystic ovaries; colour Doppler and hormonal correlations. Ultrasound Obstet Gynecol 1998 May;11(5):332-336.

22. Battalgia C, Artini PG, D'Ambrogio G, Genazzani AD, Genazzani AR. The role of colour Doppler imaging in the diagnosis of polycystic ovarian syndrome. Am J Obstet Gynecol 1995 Jan;172(1 Pt 1):108-113.

23. Lam PM, Jhonson IR, Rainne-Fenning NJ. Three dimensional ultrasound features of the polycystic ovary and the effect of different phenotypic expressions on these parameters. Hum Reprod 2007 Dec;22(12):3116-3123.

24. Legro RS, Chui P, Kunselman AR, Bentley CM, Dodson WC, Dunaif A. Polycystic ovaries are common in women with hyperandrogenic chronic anovulation but do not predict metabolic or reproductive genotype. J Clin Endocrinol Metab 2005 May;90(5):2571-2579.

25. Buckett WM, Bouzayen R, Watkin KL, Tulandi T, Tan SL. Ovarian stromal echogenicity in women with normal and polycystic ovaries. Hum Reprod 1999 Mar;14(3):618-621.
26. Franks S, Webber LJ, Micaela Goh, Valentine A, White DM, Gerard S, Conway GS, Wiltshire S, McCarthy MI. Ovarian morphology is a marker of heritable biochemical traits in sisters with polycystic ovaries. J Clin Endocrinol Metab 2008 Sep;93(9):3396-3402.

27. Lam PM, Raine-Fenning N. The role of three dimensional ultrasonography in polycystic ovary syndrome. Hum Reprod 2006 Sep;21(9):2209-2215.

28. Fevold HL. Synergism of follicle stimulating and luteinizing hormone in producing oestrogen secretion. J Clin Endocrinol Metab 1941;21.

29. Short RV. Steroids in the follicular fluid and the corpus luteum of the mare. A "two-cell type" theory of ovarian steroid synthesis. J Endocrinol 1962 Mar;24:59-63.

30. Jokubkeine L, Sladkevicius P, Rovas L, Valentine L. Assessment of changes in volume and vascularity of ovaries during the normal menstrual cycle using three dimensional power Doppler ultrasound. Hum Reprod 2006 Jun;21(10):2661-2668.

31. Campbell S, Bourne T, Waterstone J, Reynolds K, Crayford T, Jurkovic D, Okokon E, Collins W. Transvaginal color blood flow imaging of the preovulatory follicle. Fertil Steril 1993;60(3): 433-438.

32. Kupesic S, Kurjak A. Uterine and ovarian perfusion during the periovulatory period assessed by transvaginal color Doppler. Fertil Steril 1993;60:439-443.

33. Bourne TH, Jurkovic D, Waterstone J, Campbell S, Collins WP. Intrafollicular blood flow during human ovulation, Ultrasound Obstet Gynecol 1991 Jan;1(1):53.

34. Tan SL, Zaidi J, Campbell S, Doyle P, Collins W. Blood flow changes in the ovarian and uterine arteries during the normal menstrual cycle. Am J Obstet Gynecol 1996 Sep;175(3 Pt 1): 625-631.

35. Bassil S, Magritte JP, Roth J, Nisolle M, Donnez J, Gordts S. Uterine vascularity during stimulation and its correlation with implantation in IVF. Hum Reprod 1995 Jun;10(6):1497-1501.

36. Ng EH, Chan CC, Tang OS, Yeung WS, Ho PC. Relationship between uterine blood flow and endometrial and subendometrial blood flows during stimulated and natural cycles. Fertil Steril 2006 Mar;85(3):721-727.

37. Chui D, Pugh N, Walker S, Gregory L, Shaw R. Follicular vascularity - the predictive value of transvaginal power Doppler ultrasonography in an in vitro fertilization program: a preliminary study. Hum Reprod 1997 Jan;12(1):191-196.

38. Nargund G, Doyle PE, Bourne TH, Parsons JH, Cheng WC, Campbell S, Collins WP. Ultrasound-device indices of follicular blood flow before HCG administration and prediction of oocyte recovery and preimplantation embryo quality. Hum Reprod 1996 Nov;11(11):2512-2517.

39. Nargund G, Bourne TH, Doyle PE, Parsons J, Cheng W, Campbell S, Collins W. Association between ultrasound indices of follicular blood flow, oocyte recovery and preimplantation embryo quality. Hum Reprod 1996 Jan;11(1):109-113.

40. Van Blerkom J, Davis P, Alexander S. Inner mitochondrial membrane potential, cytoplasmic ATp content and free $\mathrm{Ca}^{2+}$ levels in metaphase II mouse oocytes. Hum Reprod 2003 Nov;18(11):2429-2440.

41. Smith B, Porter R, Ahuja K, Craft I. Ultrasonic assessment of endometrial changes in stimulated cycles in an in vitro fertilization and embryo transfer program. J In Vitro Fert Embryo Transf 1984 Dec;1(4):233-238.

42. Dogan MM, Uygur D, Alkan RN, Batioglu S, Mollamahmutoglu L. Prediction of pituitary down-regulation 
by evaluation of endometrial thickness in an IVF programme. Reprod Biomed Online 2004 May;8(5):595-599.

43. Zaidi J, Campbell S, Pittrof FR, Tan SL. Endometrial thickness morphology, vascular penetration and velocimetry in predicting implantation in an IVF program. Ultrasound Obstet Gynecol 1995 Sep;6(3):191-198.

44. Applebaum M. The "steel" and "teflon" endometriumultrasound visualization of endometrial vascularity in IVF patients and outcome. Presented at The Third World Congress of Ultrasound in Obstetrics and Gynecology. Ultrasound Obstet Gynecol 1993;3(Suppl 2):10.

45. Yang J-H, Wu MY, Chen CD, Jiang MC, Ho HN, Yang YS. Association of endometrial blood flow as determined by a modified colour Doppler technique with subsequent outcome of IVF. Hum Reprod 1999 Jun;14(6):1606-1610.

46. Chein LW, Au HK, Chen PL, Xiao J, Tzeng CR. Assessment of uterine receptivity by the endometrial-subendometrial blood flow distribution pattern in women undergoing IVF-ET. Fertil Steril 2002 Aug;78(2):245-245.
47. Coulam CB, Stern JJ, Soenksen DM, Britten S, Bustillo M. Comparison of pulsatility indexes on the day of oocyte retrieval and embryo transfer. Hum Reprod 1995 Jan;10(1):82-84.

48. Tsai Y-C, Chang J-C, Tai M-J, Kung F-T, Yang L-C, Chang S-Y. Relationship of uterine perfusion to outcome of intrauterine insemination. J Ultrasound Med 1996 Jul-Sep;15(9):633-636.

49. Cacciatore B, Simberg N, Fusaro P, Tiitinen A. Transvaginal Doppler study of uterine artery blood flow in in vitro fertilization embryo transfer cycles. Fertil Steril 1996 Jul;66(1):130-134.

50. Salle B, Bied-Damon V, Benchaib M, Desperes S, Gaucherand P, Rudigoz RC. Preliminary report of an ultrasonography and colour Doppler uterine score to predict uterine receptivity in an IVF programme. Hum Reprod 1998 Jun;13(6):1669-1673.

51. Kupesić S, Kurjak A, Vujisić S, Petrović Z. Luteal phase defect: comparison between Doppler velocimetry, histological and hormonal markers. Ultrasound Obstet Gynecol 1997 Feb;9(2):105-112.

52. Glock JL, Brumsted JR. Colour flow pulsed Doppler ultrasound in diagnosing luteal phase defect. Fertil Steril 1995 Sep;64(3):500-504. 